\title{
LMI-based Design of State-Derivative Feedback Control for Takagi-Sugeno Fuzzy Descriptor Systems
}

\author{
Elvis Mayk Chaves Barbosa* Fernando de Oliveira Souza** \\ Reinaldo Martinez Palhares ** \\ * Graduate Program in Electrical Engineering - Universidade Federal \\ de Minas Gerais - Av. Antônio Carlos 6627, 31270-901, Belo \\ Horizonte, $M G$, Brazil, \\ (e-mail: elvismayk@ufmg.br). \\ ** Department of Electronics Engineering - Universidade Federal de \\ Minas Gerais - Av. Antônio Carlos 6627, 31270-901, Belo Horizonte, \\ MG,Brazil(e-mails: fosouza@cpdee.ufmg.br; rpalhares@ufmg.br)
}

\begin{abstract}
This paper is concerned to present design conditions for state-derivative feedback control for Takagi-Sugeno fuzzy descriptor models. The main contribution is to derive a PDC control law without the requirement to rewrite the fuzzy model to fit in some specific format. We propose sufficient conditions as Linear Matrices Inequalities with or without the decay rate constraint in the trajectories, assuming that all eigenvalues of the fuzzy plant models are notnull. Three examples illustrate the effectiveness the proposed conditions to derive gains for the state-derivative control law to stabilize nonlinear descriptor systems.
\end{abstract}

Keywords: Descriptor systems, Takagi-Sugeno models, State-derivative feedback, Linear Matrix Inequalities.

\section{INTRODUCTION}

As a matter of fact, descriptor systems have been investigated extensively since the seminal paper of Luenberger (1978) and, from the modeling point of view, this kind of system description (i.e., a differential algebraic system) arises naturally in a wide variety of real problems. We can cite the use of descriptor systems to modeling mechanical systems (Blandeau et al., 2018; Guelton et al., 2008), robots (Schulte and Guelton, 2009) and electrical circuits (Newcomb and Dziurla, 1989).

In the context of nonlinear systems, the Tagaki-Sugeno fuzzy (T-S) modeling has become popular to represent nonlinear dynamics by a combination of linear systems (Tanaka and Wang, 2001). Nguyen et al., 2019 present a review about the history and point out the perspective of Fuzzy Control Systems. The first statement of T-S descriptor fuzzy system was made in Taniguchi et al. (1999) and its results were extended in Taniguchi et al. (2000).

In some practical situations, the state-derivative is easier to obtain than the state signals (Faria et al., 2010b). Abdelaziz and Valášek (2004) presented pole-placement technique for state-derivative feedback in SISO linear systems. Faria et al. (2009) presented LMI conditions to design a state-derivative feedback for linear multi-variable systems. In da Silva et al. (2011) the work of Faria et al. (2007), which presented LMI conditions to design stabilizing control T-S fuzzy standard systems, is generalized to uncertain T-S fuzzy standard systems. Further Kaewpraek and Assawinchaichote (2017) added the $H_{\infty}$ performance. Moreover, Faria et al. (2010a) dealt with state-derivative feedback in descriptor systems subject to structural failures in the plant.

A survey about derivative feedback control for descriptor systems is given by Junchao et al. (2006), which stated that the derivative feedback could change the derivative matrix for regularizing and controlling purposes. However, to the best of the authors knowledge, the stabilization problem for T-S fuzzy descriptor models using state-derivative feedback has not been investigated fully up to now.

The main purpose of this paper is to propose new LMI conditions for the design of a PDC (Parallel distributed Compensation) control law with a state-derivative feedback to improve the stabilization of T-S fuzzy descriptor models. The main idea is to achieve better performance in some sense for the nonlinear closed-loop system.

The paper is organized as follows: in Section 2 is presented the notation and the theoretical foundation around the technique of state-derivative feedback presented in Section 3. In order to show the effectiveness of the proposed controller design, numerical application examples are presented in Section 4. Finally, Section 5 concludes this work.

\section{PRELIMINARIES AND NOTATION}

Consider the following continuous-time controllable nonlinear descriptor model of the form:

$$
E(x(t)) \dot{x}(t)=A(x(t)) x(t)+B(x(t)) u(t)
$$


where $x(t)=\left[x_{1}, \ldots, x_{n}\right]^{T} \in \mathbb{R}^{n}$ is the descriptor vector, $u(t)=\left[u_{1}, \ldots, u_{m}\right]^{T} \in \mathbb{R}^{m}$ is the input vector, $E(x(t)) \in \mathbb{R}^{n \times n}, A(x(t)) \in \mathbb{R}^{n \times n}$ and $B(x(t)) \in \mathbb{R}^{n \times m}$. The uniqueness and existence of solutions for system $(E(x(t)), A(x(t)), B(x(t)))$ is guaranteed if $\operatorname{det}(\lambda E-A) \neq$ 0 for some $\lambda \in \mathbb{C}$ and, then the pair $(E, A)$ is called regular (Dai, 1989). The existing solution is impulsive-free if $\operatorname{deg}(\operatorname{det}(\lambda E-A))=\operatorname{rank}(E)$, for any initial condition. The system (1) could be represented by the following T-S fuzzy model defined in Taniguchi et al. (2000):

$$
\sum_{k=1}^{r_{e}} v_{k}(z(t)) E_{k} \dot{x}(t)=\sum_{i=1}^{r_{a}} h_{i}(z(t))\left(A_{i} x(t)+B_{i} u(t)\right)
$$

where $E_{k} \in \mathbb{R}^{n \times n}, A_{i} \in \mathbb{R}^{n \times n}$ and $B_{i} \in \mathbb{R}^{n \times m}$ are constant matrices. $z(t)$ is the vector of premise variables and each $v_{k}(z(t))$ and $h_{i}(z(t))$ is a membership function (MF) ensuring the following convex-sum property:

$$
\begin{array}{lll}
v_{k}(z(t))>0, & \sum_{k=1}^{r_{e}} v_{k}(z(t))=1 \\
h_{i}(z(t))>0, & \sum_{i=1}^{r_{a}} h_{i}(z(t))=1
\end{array}
$$

In order to simplify the notation, we rewrite (2) as:

$$
E_{v} \dot{x}=A_{h} x+B_{h} u
$$

where

$$
\begin{aligned}
A_{h} & =\sum_{i=1}^{r_{a}} h_{i}(z(t)) A_{i} \quad B_{h}=\sum_{i=1}^{r_{a}} h_{i}(z(t)) B_{i} \\
E_{v} & =\sum_{k=1}^{r_{e}} v_{k}(z(t)) E_{k}
\end{aligned}
$$

This work is intended to find constant matrices $K_{i k} \in$ $\mathbb{R}^{m \times n}$ with $i=1, \ldots, r_{a}$ and $k=1, \ldots, r_{e}$ to compute a PDC control law of the form

$$
u(t)=-\sum_{i=1}^{r_{a}} \sum_{k=1}^{r_{e}} h_{i}(z) v_{k}(z) K_{i k} \dot{x}(t)=-K_{v h} \dot{x}
$$

such that such (2) with the PDC control law in (4) given by:

$$
\begin{aligned}
& E_{v} \dot{x}=A_{h} x(t)-B_{h} K_{v h} \dot{x} \Rightarrow \\
& \dot{x}=\left(E_{v}+B_{h} K_{v h}\right)^{-1} A_{h} x
\end{aligned}
$$

be regular, impulsive-free, asymptotically stable and the following condition holds:

$$
\operatorname{det}\left(E_{k}+B_{i} K_{j k}\right) \neq 0
$$

for $i, j \in\left\{1, \ldots, r_{a}\right\}$ and $k \in\left\{1, \ldots, r_{e}\right\}$. We suppose that the matrices $A_{i}$ with $i=1, \ldots, r_{a}$ are nonsingular $\left(\operatorname{det}\left(A_{i}\right) \neq 0, \forall i\right)$. From this point, the time $t$, argument of the signals, and the premise variable $z$ of the MF, is omitted.

Remark 1. According with Duan et al. (1999) its assured that $(E+B K)$ is a nonsingular matrix only if:

$$
\operatorname{rank}[E, B]=n
$$

\subsection{Useful Results}

Consider a controllable linear descriptor system of the form

$$
E \dot{x}=A x+B u
$$

and a linear state-derivative feedback control law given as

$$
u=-K \dot{x}
$$

with $K \in \mathbb{R}^{m \times n}$. Assuming that $(E+B K)$ is nonsingular, the closed-loop of the system (8) with (9) can be written as

$$
\dot{x}=(E+B K)^{-1} A x(t)
$$

In this way, the following theorem, based on a quadratic Lyapunov function, is very useful.

Theorem 2. (Faria et al., 2010a). Assuming that (7) holds, a necessary and sufficient condition to the system (10) be globally asymptotically stable is the existence of matrices $Q=Q^{T} \in \mathbb{R}^{n \times n}$ and $Y \in \mathbb{R}^{m \times n}$ such that:

$$
\begin{gathered}
A Q E^{T}+E Q A^{T}+B Y A^{T}+A Y^{T} B^{T}<0 \\
Q>0
\end{gathered}
$$

with the state-derivative feedback matrices are given by:

$$
K=Y Q^{-1}
$$

Proof. See Faria et al. (2010a).

The Theorem 2 guarantees that if $\operatorname{det}\left(A_{i}\right) \neq 0, \forall i, A_{h}$ is nonsingular (Faria et al., 2010b).

\section{MAIN RESULTS}

Based on Theorem 2 we can state the following:

Theorem 3. The T-S fuzzy descriptor system in (5) is regular, impulsive-free and asymptotically stable, if there exist a positive-definite matrix $Q \in \mathbb{R}^{n \times n}$ and matrices $Y_{j k} \in \mathbb{R}^{m \times n}$ such that the following LMIs are satisfied for $i, j, l \in\left\{1, \ldots, r_{a}\right\}$ and $k \in\left\{1, \ldots, r_{e}\right\}$ :

$$
E_{k} Q A_{l}^{T}+A_{l} Q E_{k}^{T}+B_{i} Y_{j k} A_{l}^{T}+A_{l} Y_{j k}^{T} B_{i}^{T}<0
$$

and the state-derivative feedback matrix is given by:

$$
K_{j k}=Y_{j k} Q^{-1}
$$

Proof. Considering the convex-sum property of the MF, follows from (13) that:

$$
\begin{gathered}
\sum_{i, j, l=1}^{r_{a}} h_{i} h_{j} h_{l} \sum_{k=1}^{r_{e}} v_{k}\left[E_{k} Q A_{i}^{T}+A_{i} Q E_{k}^{T}+B_{i} Y_{j k} A_{l}^{T}+A_{l} Y_{j k}^{T} B_{i}^{T}\right]= \\
=E_{v} Q A_{h}^{T}+A_{h} Q E_{v}^{T}+B_{h} Y_{h v} A_{h}^{T}+A_{h} Y_{h v}^{T} B_{h}^{T}<0
\end{gathered}
$$

where

$$
Y_{v h}=\sum_{j=1}^{r_{a}} \sum_{k=1}^{r_{e}} h_{j} v_{k} Y_{j k}
$$

Thus, we have the same condition of Theorem 2, with $E=E_{v}, A=A_{h}, B=B_{h}$ and $Y=Y_{v k}$. Therefore, the existence of matrices $Q$ and $Y_{j k}$ such that (13) holds is a sufficient condition for the asymptotically stability of system (5).

Notice that the proposed LMI conditions do not require to rewrite the T-S fuzzy descriptor model unlike the approach proposed in Taniguchi et al. (2000) and directly do not derive matching conditions in results (Taniguchi et al. (1999)). In these designs, have the derivative matrix a singular derivative $E$ in the closed-loop fuzzy model, which implies the necessity to prove the regularity and the exemption impulsive responses.

The reason for not needing to rewrite the model for the control design in our approach is because the statederivative feedback regularizes the derivative matrix of the closed-loop system as can be seen in (5). 
According with Boyd et al. (1994), the decay rate is defined as the largest $\gamma$ such that:

$$
\lim _{t \rightarrow \infty} e^{\gamma t}|| x(t) \|=0
$$

holds for all trajectories of $x$. Using the quadratic Lyapunov function to establish a lower bound on the decay rate, we have:

where $V(x)=x^{T} P x$.

$$
\dot{V}(x)<-2 \gamma V(x)
$$

Thus, we can state the following theorem:

Theorem 4. The T-S fuzzy descriptor system in (5) is regular, impulsive-free and asymptotically stable with a decay rate (16) greater to $\gamma>0$, if there exist a positivedefinite matrix $Q \in \mathbb{R}^{n \times n}$ and matrices $Y_{j k} \in \mathbb{R}^{m \times n}$ such that the following LMIs are satisfied for $i, j, l \in\left\{1, \ldots, r_{a}\right\}$ and $k \in\left\{1, \ldots, r_{e}\right\}$ :

$$
\left[\begin{array}{c}
E_{k} Q A_{l}^{T}+A_{l} Q E_{k}^{T}+B_{i} Y_{j k} A_{l}^{T}+A_{l} Y_{j k}^{T} B_{i}^{T} \\
Q E_{k}^{T}+Y_{j k}^{T} B_{i}^{T} \\
E_{k} Q+B_{i} Y_{j k} \\
-\frac{Q}{2 \gamma}
\end{array}\right]<0
$$

and the state-derivative feedback matrices are given by:

$$
K_{j k}=Y_{j k} Q^{-1}
$$

Proof. By (17) and computing $\dot{V}$ we have:

$$
\begin{gathered}
\dot{V}(x)=x^{T} A_{h}^{T}\left(E_{v}+B_{h} K_{h v}\right)^{-T} P x+x^{T} P\left(E_{v}+\right. \\
\left.B_{h} K_{h v}\right)^{-1} A_{h} x<-2 \gamma x^{T} P x \Rightarrow \\
A_{h}^{T}\left(E_{v}+B_{h} K_{h v}\right)^{-T} P+P\left(E_{v}+B_{h} K_{h v}\right)^{-1} A_{h}< \\
-2 \gamma P
\end{gathered}
$$

where $M^{-T}$ means the transpose of $M^{-1}$.

Pre and post-multiplying $(20)$ by $\left(E_{v}+B_{h} K_{h v}\right) P^{-1}$ and $P^{-1}\left(E_{v}+B_{h} K_{h v}\right)^{T}$, respectively, and applying the convexsum property of MF and the Schur complements (Boyd et al., 1994) we obtain:

$$
\left[\begin{array}{c}
E_{k} P^{-1} A_{i}^{T}+A_{l} P^{-1} K_{j k}^{T} B_{i}^{T}+A_{i} P^{-1} E_{k}^{T}++B_{i} K_{j k} P^{-1} A_{l}^{T} \\
P^{-1} E_{k}^{T}+P^{-1} K_{j k}^{T} B_{i}^{T} \\
E_{k} P^{-1}+B_{i} K_{j k} P^{-1} \\
-\frac{P^{-1}}{2 \gamma}
\end{array}\right]<0
$$

Defining $Q=P^{-1}$ and $Y_{j k}=K_{j k} P^{-1}$, then it follows the LMI condition (18). Therefore, given a $\gamma>0$, the existence of matrices $Q$ and $Y_{j k}$ such that (18) holds is a sufficient condition for the asymptotically stability of system (5) with a decay rate greater than $\gamma$.

\section{NUMERICAL EXAMPLES}

The numerical examples were simulated using Matlab. The computational implementations of the LMI conditions have been carried out using as parser and solver, YALMIP and Mosek 8, respectively.

\subsection{Example 1}

Consider the following system from Taniguchi et al. (2000) which nonlinear dynamics described by:

$$
(1+a \cos (\theta(t))) \ddot{\theta}(t)=-b \dot{\theta}^{3}(t)+c \theta(t)+d u(t)
$$

where it is assumed that $a<1$ and $|\dot{\theta}(t)|<\phi$. We use $a=0.2, b=1, c=-1, d=10$ and $\phi=4$. The nonlinear system in (21) can be exactly represented by the following fuzzy descriptor system:

$$
\sum_{k=1}^{2} v_{k}\left(x_{1}(t)\right) E_{k} \dot{x}=\sum_{i=1}^{2} h_{i}\left(x_{2}(t)\right)\left(A_{i} x(t)+B_{i} u(t)\right)
$$

where $x(t)=\left[x_{1}(t) x_{2}(t)\right]^{T}=[\theta(t) \dot{\theta}(t)]^{T}$,

$$
\begin{array}{rlr}
E_{1} & =\left[\begin{array}{cc}
1 & 0 \\
0 & 1+a
\end{array}\right], & E_{2}=\left[\begin{array}{cc}
1 & 0 \\
0 & 1-a
\end{array}\right], \\
A_{1}=\left[\begin{array}{cc}
0 & 1 \\
c & -b \phi^{2}
\end{array}\right], & A_{2}=\left[\begin{array}{ll}
0 & 1 \\
c & 0
\end{array}\right], \\
B_{1}=B_{2}=\left[\begin{array}{l}
0 \\
d
\end{array}\right] & &
\end{array}
$$

and the membership functions

$$
\begin{array}{ll}
h_{1}\left(x_{2}\right)=\frac{x_{2}^{2}}{\phi^{2}} & h_{2}\left(x_{2}\right)=1-h_{1}\left(x_{2}\right) \\
v_{1}\left(x_{1}\right)=\frac{1+\cos \left(x_{1}\right)}{2} & v_{2}\left(x_{1}\right)=1-v_{1}\left(x_{1}\right)
\end{array}
$$

Notice that the derivative matrices, $E_{1}$ and $E_{2}$, are regular and positive definite.

Applying Theorem 3, we find that the LMIs condition are feasible with:

$$
\begin{aligned}
& Q=\left[\begin{array}{cc}
0.2627 & -0.0314 \\
-0.0314 & 1.0037
\end{array}\right] \\
& Y_{11}=Y_{21}=\left[\begin{array}{ll}
0.1048 & -0.1207
\end{array}\right] \\
& Y_{12}=Y_{22}=\left[\begin{array}{ll}
0.1035 & -0.0805
\end{array}\right]
\end{aligned}
$$

and the PDC control gains in (4) are calculated using (14):

$$
\begin{aligned}
& K_{11}=K_{12}=\left[\begin{array}{ll}
0.3860 & -0.1082
\end{array}\right] \\
& K_{21}=K_{22}=\left[\begin{array}{ll}
0.3860 & -0.0682
\end{array}\right]
\end{aligned}
$$

Figure 1 depicts the trajectories of the states and the input control of the closed-loop system with (4) (with gain given in (23)) and the exact model in (21), that is, using the original system for the simulation not the T-S fuzzy model in (22). Notice that the T-S fuzzy model in (22) was used only to compute the control gains via Theorem 3 . In this case we intend to show the effectiveness of the PDC control design proposed, i.e., the PDC controller is tested to control directly the original system in (16), without any kind of approximation. In Figure 1 was used as initial condition $x_{0}=\left[\begin{array}{ll}2 & -0.5\end{array}\right]^{T}$.

\subsection{Example 2}

The system in (21) can also be exactly represented by the following fuzzy descriptor form given in Lin et al. (2006):

$$
E \dot{x}=\sum_{i=1}^{3} h_{i}(x(t))\left(A_{i} x(t)+B_{i} u(t)\right)
$$

where $x(t)=\left[\begin{array}{lll}x_{1}(t) & x_{2}(t) x_{3}(t)\end{array}\right]^{T}=\left[\begin{array}{lll}\theta(t) & \dot{\theta}(t) \ddot{\theta}(t)\end{array}\right]^{T}$

$E=\left[\begin{array}{lll}1 & 0 & 0 \\ 0 & 1 & 0 \\ 0 & 0 & 0\end{array}\right] \quad A_{1}=\left[\begin{array}{ccc}0 & 1 & 0 \\ 0 & 0 & 1 \\ c & -b\left(\phi^{2}+2\right) & (a-1)\end{array}\right]$

$A_{3}=\left[\begin{array}{llc}0 & 1 & 0 \\ 0 & 0 & 1 \\ c & 0 & (a-1)\end{array}\right]$

$A_{2}=\left[\begin{array}{llc}0 & 1 & 0 \\ 0 & 0 & 1 \\ c & 0 & \left(-a-1-a \phi^{2}\right)\end{array}\right]$

$B_{1}=B_{2}=B_{3}=\left[\begin{array}{l}0 \\ 0 \\ d\end{array}\right]$ 


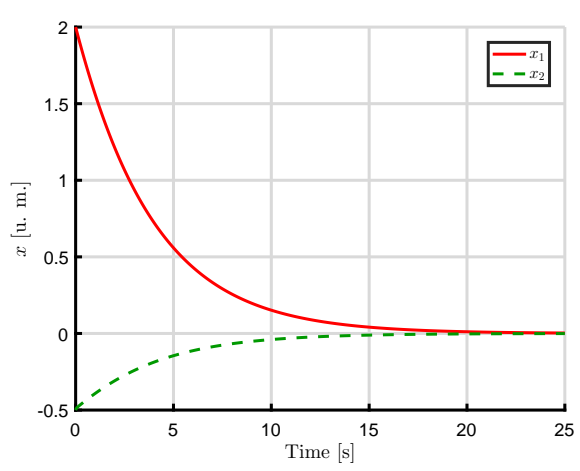

(a) $x(t)$

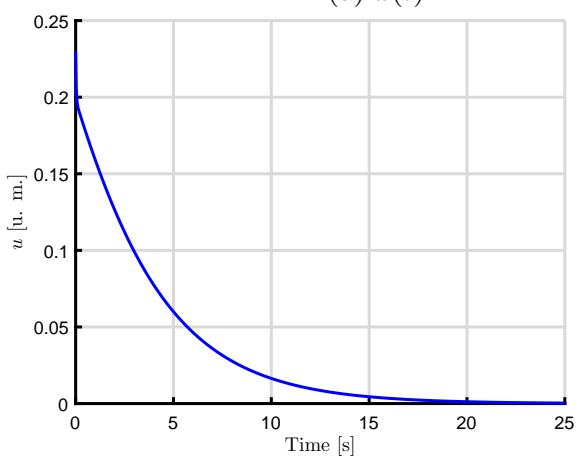

(b) $u(t)$

Figure 1. Trajectories for the closed-loop system with (21) - the original nonlinear sysytem - and the statederivative feedback as in (4) with gain obtained in (23) for initial condition $x_{0}=[2-0.5]^{T}$. Example 1 .

and the MF are defined as following:

$$
\begin{aligned}
h_{1} & =\frac{x_{2}^{2}}{\phi^{2}+2} & h_{2}=\frac{1+\cos \left(x_{1}\right)}{\phi^{2}+2} \\
h_{3} & =\frac{\phi^{2}-x_{2}^{2}+1-\cos \left(x_{1}\right)}{\phi^{2}+2} &
\end{aligned}
$$

holding the convex-sum property. Notice that the derivative matrix is singular and the condition given in Remark 1 holds.

Applying the conditions stated in Theorem 3, we get the following solution

$$
\begin{aligned}
& Q=\left[\begin{array}{rrr}
0.2251 & -0.0271 & -0.0262 \\
-0.0271 & 0.0208 & -0.1645 \\
-0.0262 & -0.1645 & 2.3286
\end{array}\right] \\
& Y_{11}=Y_{21}=Y_{31}=\left[\begin{array}{lll}
0.1562 & -0.0002 & 0.0117
\end{array}\right]
\end{aligned}
$$

which results the PDC control gains for (4) using (14):

$$
K_{11}=K_{21}=K_{31}=\left[\begin{array}{lll}
1.396 & 3.4638 & 0.2525
\end{array}\right]
$$

The results of the simulation of (21) in closed-loop with control law (4) and gains given in (25) for initial condition $x_{0}=\left[\begin{array}{lll}2 & -0.5 & -0.2227\end{array}\right]^{T}$ are depicted in Figure 2. Following the same steps in Example 1, the T-S fuzzy model in (24) was used only to compute the state-derivative matrix via Theorem 3 , but the simulation was performed using the original nonlinear system described in (21). In this case, the idea is to illustrate the effectiveness of the proposed approach, but ensuring condition stated in Remark 1, for the case when $E$ is not full rank.

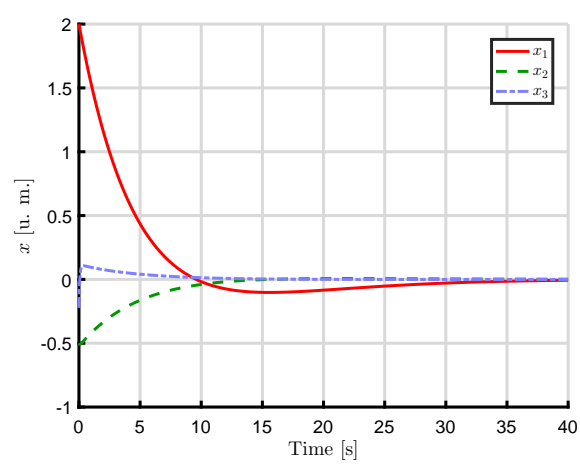

(a) $x(t)$

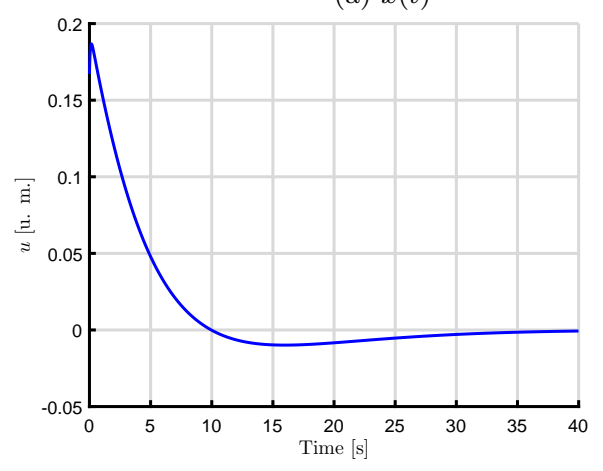

(b) $u(t)$

Figure 2. Trajectories for the closed-loop system with (21) - the original nonlinear sysytem - and the statederivative feedback as in (4) with gain obtained in (25) for initial condition $x_{0}=\left[\begin{array}{lll}2 & -0.5 & -0.2227\end{array}\right]^{T}$. Example 2.

\subsection{Example 3}

The third example is borrowed from Blandeau et al. (2016) to ilustrate the effectiveness of the proposed approach in a system with $n=4$. Consider a nonlinear system as in (1) where:

$$
\begin{gathered}
E(x)=\left[\begin{array}{cccc}
1 & 0 & 0 & 0 \\
0 & m_{1} & 0 & -m_{1} l_{0} \\
0 & 0 & 1 & 0 \\
0 & -m_{1} l_{0} & 0 & J\left(x_{l}\right)
\end{array}\right], \quad B(x)=\left[\begin{array}{l}
0 \\
1 \\
0 \\
0
\end{array}\right], \\
A(x)=\left[\begin{array}{cccc}
0 & 1 & 0 & 0 \\
0 & 0 & -m_{1} g \frac{\sin \theta}{\theta} & m_{1} x_{l} \dot{\theta} \\
0 & 0 & 0 & 1 \\
-m_{1} g \cos \theta & -2 m_{1} x_{l} \dot{\theta} & \left(\begin{array}{c}
m_{1} l_{0}+ \\
m_{2} l_{c}
\end{array}\right) g \frac{\sin \theta}{\theta} & 0
\end{array}\right]
\end{gathered}
$$

with $J\left(x_{l}\right)=\left(m_{1}\left(l_{0}^{2}+x_{l}^{2}\right)+m_{2} l_{c}^{2}\right)$.

This model represents the dynamics of the H2AT ("head, two arms and trunk") pendulum, that is an extended version of the planar inverted pendulum consisting of two rods and it is used to study sitting stability of persons with Spine Cord Injury (Blandeau et al., 2016).

The parameters are: $m_{1}=16.1 \mathrm{~kg}, m_{2}=26.64 \mathrm{~kg}, l_{0}=$ $477 \mathrm{~mm}, l_{c}=276.66 \mathrm{~mm}, g=9.81 \mathrm{~m} / \mathrm{s}^{2}$. The operation ranges for the states of the system are presented in the following compact set:

$$
\Omega=\left\{\begin{array}{c}
\underline{x}_{l}=-0.07518 \leq x_{l} \leq 0.10527=\bar{x}_{l} \\
\underline{\theta}=-0.175 \leq \theta \leq 0.349=\bar{\theta} \\
\underline{\dot{\theta}}=-0.5 \leq \dot{\theta} \leq 0.5=\overline{\dot{\theta}}
\end{array}\right\}
$$


Blandeau et al. (2018) propose to consider $\sin (\theta) / \theta \approx$ $\cos (\theta)$ for the operational range presented in $(27)$, then the H2AT can be approximately represented by:

$$
\sum_{k=1}^{2} v_{k} E_{k} \dot{x}=\sum_{i=1}^{4} h_{i} A_{i} x(t)+B u(t)
$$

where

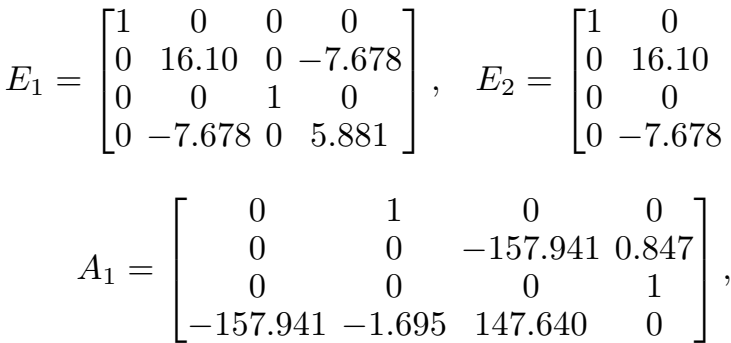

$$
\begin{aligned}
& A_{2}=\left[\begin{array}{cccc}
0 & 1 & 0 & 0 \\
0 & 0 & -157.941 & -0.847 \\
0 & 0 & 0 & 1 \\
-157.941 & 1.695 & 147.640 & 0
\end{array}\right] \text {, } \\
& A_{3}=\left[\begin{array}{cccc}
0 & 1 & 0 & 0 \\
0 & 0 & -148.420 & 0.847 \\
0 & 0 & 0 & 1 \\
-148.420 & -1.695 & 138.739 & 0
\end{array}\right] \text {, } \\
& A_{4}=\left[\begin{array}{cccc}
0 & 1 & 0 & 0 \\
0 & 0 & -148.420 & -0.847 \\
0 & 0 & 0 & 1 \\
-148.420 & 1.695 & 138.739 & 0
\end{array}\right] \text {. }
\end{aligned}
$$

The MF $h_{i}, i \in 1,2,3,4$ are constructed with the following weighting functions $(\mathrm{WF})$ :

$$
\begin{aligned}
w_{0}^{1} & =\frac{\cos (\theta)-\cos (\bar{\theta})}{\cos (0)-\cos (\bar{\theta})} & w_{1}^{1} & =1-w_{1}^{0} \\
w_{2}^{0} & =\frac{x_{l} \dot{\theta}-\bar{x}_{l} \underline{\dot{\theta}}}{\bar{x}_{l} \overline{\dot{\theta}}-\bar{x}_{l} \dot{\theta}} & w_{2}^{1} & =1-w_{2}^{0}
\end{aligned}
$$

Thus we have as MF for (28):

$$
\begin{array}{ll}
v_{1}=\frac{x_{l}^{2}}{\bar{x}_{l}^{2}} & v_{2}=1-v_{1} \\
h_{1}=w_{0}^{1} w_{0}^{2} & h_{2}=w_{0}^{1} w_{1}^{2} \\
h_{3}=w_{1}^{1} w_{0}^{2} & h_{4}=w_{1}^{1} w_{1}^{2}
\end{array}
$$

Applying Theorem 3 to the approximated model in (28), we obtain:

$$
\begin{array}{r}
Q=\left[\begin{array}{crrr}
0.0011 & -0.0027 & 0.0012 & -0.0034 \\
-0.0027 & 0.0720 & -0.0030 & 0.0919 \\
0.0012 & -0.0030 & 0.0016 & -0.0045 \\
-0.0034 & 0.0919 & -0.0045 & 0.1210
\end{array}\right], \\
Y_{j 1}=\left[\begin{array}{lrrr}
0.0291 & -0.2915 & 0.0236 & -0.3494
\end{array}\right], \\
Y_{j 2}=\left[\begin{array}{lrrr}
0.0277 & -0.2892 & 0.0225 & -0.3436
\end{array}\right], \\
j \in\{1,2,3,4\}
\end{array}
$$

and the PDC control gains in (4) are calculated using (14):

$$
\begin{aligned}
& K_{j 1}=\left[\begin{array}{llll}
2.1590 & 0.4881 & -1.8783 & -0.3798
\end{array}\right] \times 10^{4}, \\
& K_{j 2}=\left[\begin{array}{llll}
1.6592 & 0.3747 & -1.4433 & -0.2916
\end{array}\right] \times 10^{4} \text {, } \\
& j \in\{1,2,3,4\}
\end{aligned}
$$

Applying Theorem 4 with $\gamma=1$ to the approximated model in (28), that LMIs condition are feasible with:

$$
\begin{array}{r}
Q=\left[\begin{array}{rrrr}
0.0004 & -0.0011 & 0.0004 & -0.0012 \\
-0.0011 & 0.0200 & -0.0011 & 0.0252 \\
0.0004 & -0.0011 & 0.0006 & -0.0019 \\
-0.0012 & 0.0252 & -0.0019 & 0.0350
\end{array}\right], \\
Y_{j 1}=\left[\begin{array}{lrrr}
0.0302 & -0.1539 & 0.0281 & -0.1704
\end{array}\right], \\
Y_{j 2}=\left[\begin{array}{lrrr}
0.0273 & -0.0918 & 0.0243 & -0.0881
\end{array}\right], \\
\end{array}
$$

and the PDC control gains in (4) are calculated using (19):

$$
\begin{array}{r}
K_{j 1}=\left[\begin{array}{llll}
1.5016 & 0.3213 & -1.2602 & -0.2526
\end{array}\right] \times 10^{3}, \\
K_{j 2}=\left[\begin{array}{llll}
1.5935 & 0.3438 & -1.3395 & -0.2675
\end{array}\right] \times 10^{3}, \\
\\
\quad j \in\{1,2,3,4\}
\end{array}
$$

Figure 3 depicts the trajectories of the states and the input control of the closed-loop system with the PDC control law as in (4) with gains given in (29) and in (30). It is clear that the trajectories of controller with gain (30) have a faster transient response, because it is imposed a bound in decay rate in Theorem 4 .
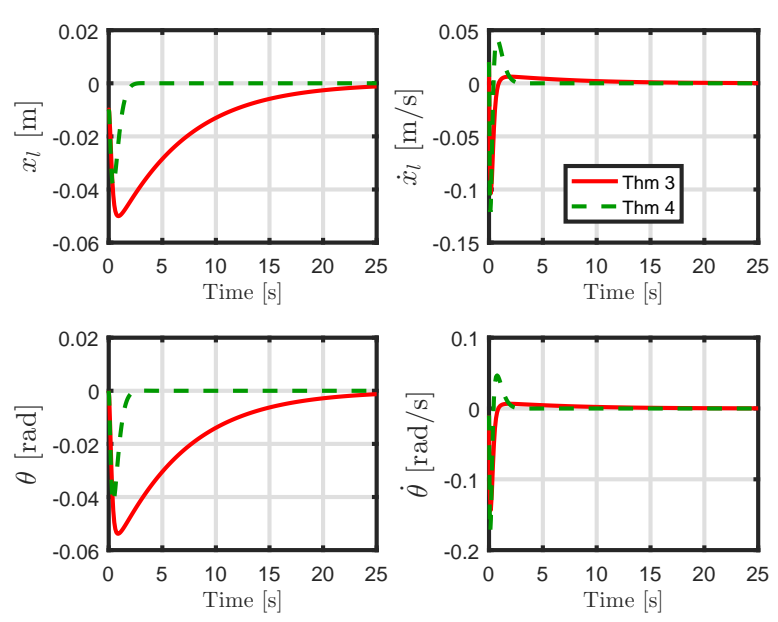

(a) $x(t)$

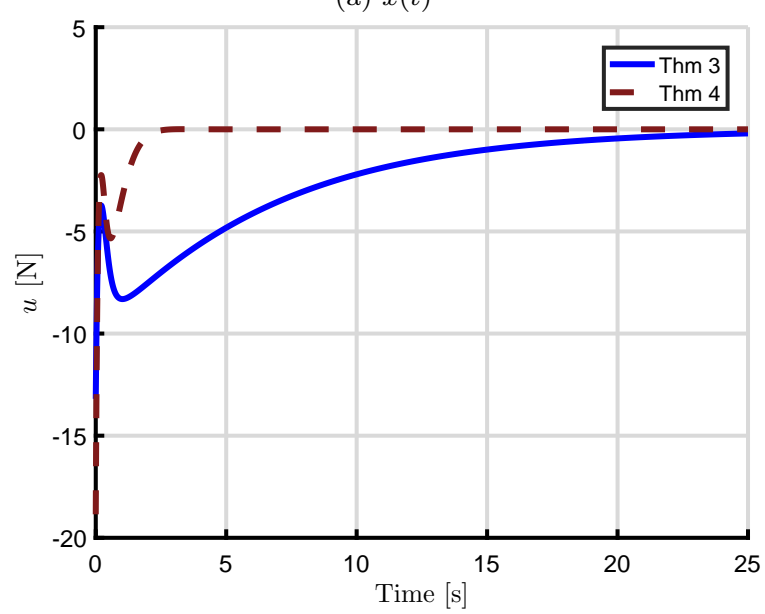

(b) $u(t)$

Figure 3. Trajectories for the closed-loop system as in (2) with (26), represented in the design by (28), with the derivative-state feedback as in (4) and gains given in (29) (Thm 3) and (30) (Thm 4) for initial condition $x_{0}=\left[\begin{array}{llll}-0.01 & 0.02 & 0 & -0.01\end{array}\right]^{T}$. Example 3 . 


\section{CONCLUSION}

This paper has proposed LMI conditions to design a PDC control law based on state-derivative feedback. These conditions require the eigenvalues of matrix $A$ are not null but it is not necessary to rewrite the system to design the controller. Numerical examples have been presented to illustrate the utility of the proposed method under different types of nonlinear descriptor models. We can cite as open problems for future works, the development of more relaxed conditions and its application in delayed T-S fuzzy descriptor systems.

\section{ACKNOWLEDGMENTS}

This work has been supported by the Brazilian agencies CAPES, CNPq and FAPEMIG.

\section{REFERENCES}

Abdelaziz, T. and Valášek, M. (2004). Pole-placement for SISO linear systems by state-derivative feedback. IEE Proceedings - Control Theory and Applications, 151(4), 377-385. doi:10.1049/ip-cta:20040660.

Blandeau, M., Estrada-Manzo, V., Guerra, T., Pudlo, P., and Gabrielli, F. (2018). Fuzzy unknown input observer for understanding sitting control of persons living with spinal cord injury. Engineering Applications of Artificial Intelligence, 67, 381-389. doi:10.1016/j.engappai.2017. 09.016 .

Blandeau, M., Guerra, T., Pudlo, P., Gabrielli, F., and Estrada-Manzo, V. (2016). How a person with spinal cord injury controls a sitting situation Unknown input observer and delayed feedback control with time-varying input delay. In 2016 IEEE International Conference on Fuzzy Systems (FUZZ-IEEE). IEEE. doi:10.1109/ fuzz-ieee.2016.7737987.

Boyd, S., El Ghaoui, L., Feron, E., and Balakrishnan, V. (1994). Linear Matrix Inequalities in System and Control Theory, volume 15 of Studies in Applied Mathematics. SIAM, Philadelphia, PA, 2nd edition.

da Silva, E.R.P., Teixeira, M.C.M., Assunção, E., and Faria, F.A. (2011). Estabilização de sistemas fuzzy T-S incertos usando realimentação derivativa. SBA: Controle $\&$ Automação Sociedade Brasileira de Automática, 22(3), 273-283. doi:10.1590/s0103-17592011000300005.

Dai, L. (1989). Singular Control Systems. Springer Berlin Heidelberg, 1 edition.

Duan, G.R., Irwin, G.W., and Liu, G.P. (1999). Robust stabilization of descriptor linear systems via proportional-plus-derivative state feedback. In Proceedings of the 1999 American Control Conference (Cat. No. 99CH36251). IEEE. doi:10.1109/acc.1999.783578.

Faria, F.A., Assunção, E., Teixeira, M.C.M., and Cardim, R. (2010a). Robust state-derivative feedback LMI-based designs for linear descriptor systems. Mathematical Problems in Engineering, 2010, 1-15. doi:10.1155/2010/ 927362.

Faria, F.A., Assunção, E., and Teixeira, M.C.M. (2009). Realimentação da derivada dos estados em sistemas multivariáveis lineares usando LMIs. SBA: Controle 8 Automação Sociedade Brasileira de Automática, 20(1), 83-93. doi:10.1590/s0103-17592009000100008.
Faria, F.A., Assunção, E., Teixeira, M.C.M., and Cardim, R. (2007). Realimentação da derivada dos estados em Sistemas Fuzzy Takagi Sugeno. Anais do VIII - Simpósio Brasileiro de Automação Inteligente. URL https:// fei.edu.br/sbai/SBAI2007/docs/29542_1.pdf.

Faria, F.A., Assunção, E., Teixeira, M.C.M., Cardim, R., and da Silva, E.R.P. (2010b). Condições menos conservadoras para o projeto de controladores fuzzy usando realimentação da derivada dos estados. In Anais do Congresso Brasileiro de Automática, 4044-4049.

Guelton, K., Delprat, S., and Guerra, T.M. (2008). An alternative to inverse dynamics joint torques estimation in human stance based on a Takagi-Sugeno unknowninputs observer in the descriptor form. Control Engineering Practice, 16(12), 1414-1426. doi:10.1016/j. conengprac.2008.04.002.

Junchao, R., Qingling, Z., and Xuefeng, Z. (2006). Derivative feedback control for singular systems. In 2007 Chinese Control Conference. IEEE. doi:10.1109/chicc. 2006.4347480 .

Kaewpraek, N. and Assawinchaichote, W. (2017). $\mathcal{H}_{\infty}$ Takagi-Sugeno Fuzzy State-Derivative Feedback Control Design For Nonlinear Dynamic Systems. International Journal of Electronics and Communication Engineering. doi:10.5281/zenodo.1129959.

Lin, C., Wang, Q.G., and Lee, T.H. (2006). Stability and stabilization of a class of fuzzy time-delay descriptor systems. IEEE Transactions on Fuzzy Systems, 14(4), 542-551. doi:10.1109/tfuzz.2006.876738.

Luenberger, D.G. (1978). Time-invariant descriptor systems. Automatica, 14(5), 473-480. doi:10.1016/ 0005-1098(78)90006-7.

Newcomb, R.W. and Dziurla, B. (1989). Some circuits and systems applications of semistate theory. Circuits, Systems, and Signal Processing, 8(3), 235-260. doi:10. $1007 /$ bf01598413.

Nguyen, A.T., Taniguchi, T., Eciolaza, L., Campos, V., Palhares, R., and Sugeno, M. (2019). Fuzzy control systems: Past, present and future. IEEE Computational Intelligence Magazine, 14(1), 56-68. doi:10.1109/mci. 2018.2881644 .

Schulte, H. and Guelton, K. (2009). Descriptor modelling towards control of a two link pneumatic robot manipulator: A T-S multimodel approach. Nonlinear Analysis: Hybrid Systems, 3(2), 124-132. doi:10.1016/j.nahs.2008. 11.003.

Tanaka, K. and Wang, H.O. (2001). Fuzzy Control Systems Design and Analysis. John Wiley \& Sons, Inc. doi: 10.1002/0471224596.

Taniguchi, T., Tanaka, K., and Wang, H. (2000). Fuzzy descriptor systems and nonlinear model following control. IEEE Transactions on Fuzzy Systems, 8(4), 442452. doi:10.1109/91.868950.

Taniguchi, T., Tanaka, K., Yamafuji, K., and Wang, H. (1999). Fuzzy descriptor systems: stability analysis and design via LMIs. In Proceedings of the 1999 American Control Conference (Cat. No. 99CH36251). IEEE. doi: 10.1109/acc.1999.786165. 